Trivent Publishing

(C) The Authors, 2016

Available online at http://trivent-publishing.eu/

Engineering and Industry Series

Volume Power Systems, Energy Markets and Renewable Energy Sources in

South-Eastern Europe

\title{
A Reversible Self-assembling Light-Harvesting System Based on a Diphenylalanine - Porphyrin Motif
}

\author{
Athanassios G. Coutsolelos, ${ }^{1}$ Anna Mitraki, ${ }^{2}$ \\ Teodor Silviu Balaban ${ }^{3}$
}

${ }^{1}$ Laboratory of Bioinorganic Chemistry, Chemistry Department, University of Crete, 710 03 Heraklion, Crete, Greece, acoutsol@uoc.gr

${ }^{2}$ Department of Materials Science and Technology University of Crete and IESLFORTH Volutes Campus, 71003, Heraklion, Crete, Greece

${ }^{3}$ Aix-Marseille Univ, ISM2, CNRS UMR 6263, Chirosciences, Avenue Escadrille Normandie Niemen, Case A62, F-13397 Marseille CEDEX 20, France

\begin{abstract}
Herein we describe a very simple diphenylalanine-dipeptide construct to which a meso-tetraphenylporphyrin (TPP) has been attached and which self-assembles to fibrils, platelets or nanospheres depending on the solvent composition. The fibrils, gave intense excitonic couplets in the electronic circular dichroism (ECD) spectra. By slightly increasing the solvent polarity, these light-harvesting fibers disassemble to spherical structures with silent ECD spectra. A single crystal X-ray structure shows that a combination of hydrogen bonding with $\pi$ stacking of the porphyrin rings is responsible for the head-to-head arrangement which explains their excitonic coupling. By unraveling the exact supramolecular architecture our study may help devise artificial antenna systems with an on/off capability.

This is an Open Access article distributed in accordance with the Creative Commons Attribution Non Commercial (CC-BY-NC-ND 4.0) license, which permits others to copy or share the article, provided original work is properly cited and that this is not done for commercial purposes. Users may not remix, transform, or build upon the material and may not distribute the modified material (http://creativecommons.org/licenses/by-nc/4.0/)
\end{abstract}




\section{Keywords}

Self-assembling; dipheylalanine; porphyrin; nano-spheres; nano-fibers; chromophore

\section{Introduction}

Artificial self-assembling light-harvesting systems are of interest in dyesensitized solar cells where a large photon capture cross section can assure that efficient energy transfer occurs to a large band-gap semiconductor. Presently, all artificial antenna systems, either assembled via covalent syntheses as in elegant dendrimeric architectures, or the self-assembling BChl c mimics, lead to large architectures which are unable to be dismantled, once they were formed.[1] Selfassemblies of short peptides such as diphenylalanine $[(L) \mathrm{F}(L) \mathrm{F})]$ have been intensively studied in recent years and have shown promising luminescence or sensing applications.[2]

We have previously prepared diphenylalanine-porphyrin conjugates and have shown that these hybrid materials self-assemble upon drying into porous nanospheres.[3] By modifying the self-assembly conditions, and dissolving the porphyrin conjugates in dichloromethane which is then "injected" into a nonpolar solvent, we observed broadened, red-shifted and splitted Soret bands typical of porphyrinic J-aggregates. This porphyrin containing self-assembling antenna system can be reversibly assembled or disassembled by slightly changing only the solvent's polarity. Furthermore we synthesized the unnatural $[(D) \mathrm{F}(D) \mathrm{F}]$-porphyrin conjugate and have studied with circular dichroism the transfer of chirality from the molecular to the supramolecular level.

\section{Structure of Synthesized Compounds}

The structure of the synthesized compounds is presented in Fig. 1. All of them were prepared in high yields after the covalent attachment of Fmoc-FF-OH or Boc-FF-OH with the amino group of the porphyrin chromophore via amide coupling. 


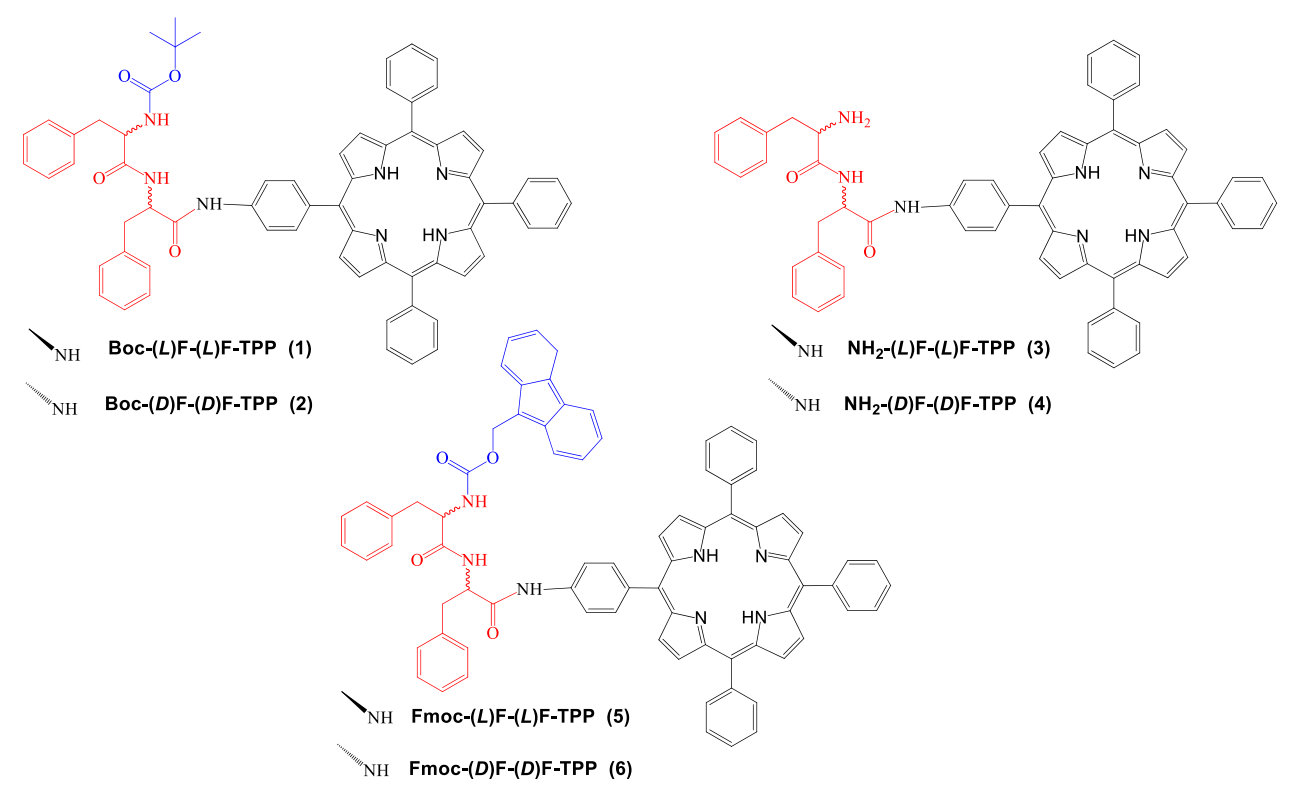

Fig. 1. The chemical structure of compounds addressed in this work

\section{Self-assembly studies in solution}

To study the properties of self-assembling porphyrinoid-amino acid bioconjugates, we performed absorption studies in solution, in the ground solid state and in the self-assembled orientation (Fig. 2). Due to the various morphologies of the nano-assemblies, we expected different spectra characteristics of the tested derivatives, as a result of their discret electronic and stereochemical properties. 


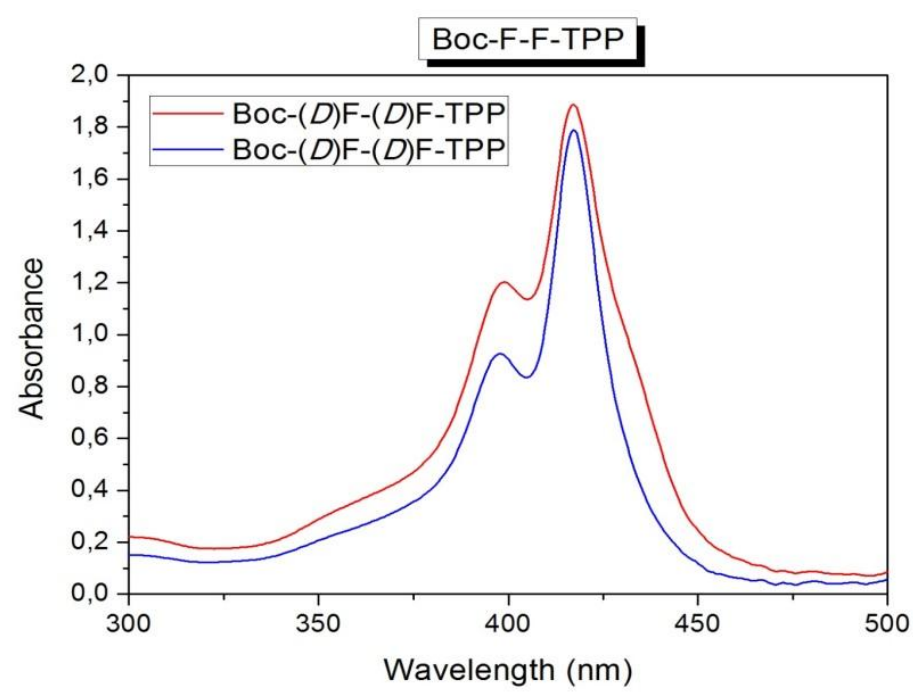

Fig. 2. The absorption spectra shows broad and split Soret bands typical of porphyrinic $\mathrm{J}$-aggregates

\section{Morphology Studies}

The diphenylalanine-porphyrin and phenylalanine-porphyrin conjugates were dissolved in various solvents, dried on glass slides, and studied by scanning electron microscopy (SEM) (Fig. 3).
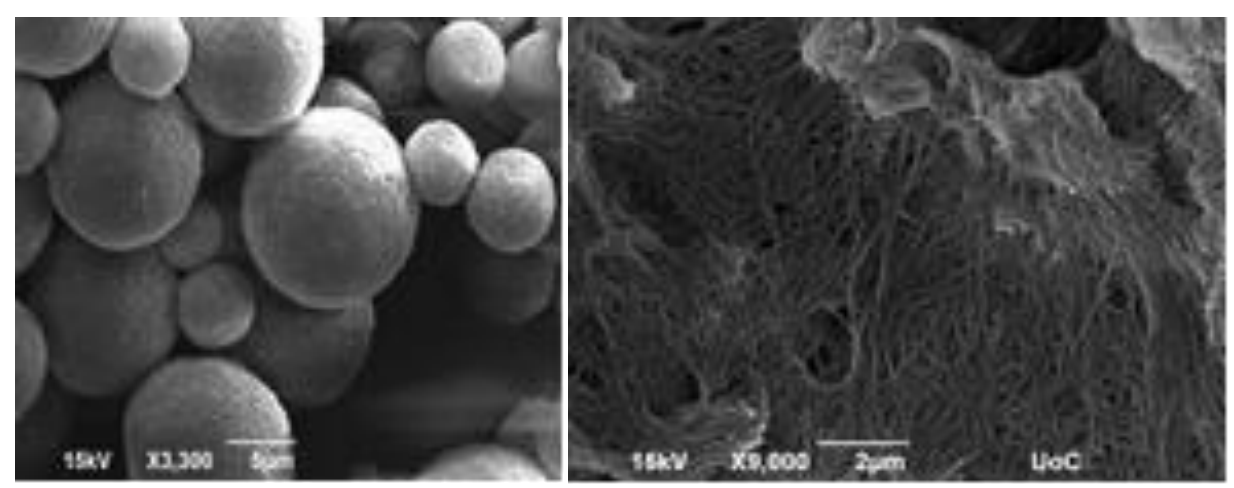

Fig. 3. Changes in the morphology with the solvent composition

\section{Circular Dichroism experiments}

Circular dichroism (CD) mesurements were performed in order to study the chirality of the nanostructures (Fig. 4). Mirror-imaged Cotton effects were 
observed for the unnatural $\mathrm{F}_{D}$ analogues, revealing that the chirality is translated from the molecular level to the superamolecular.
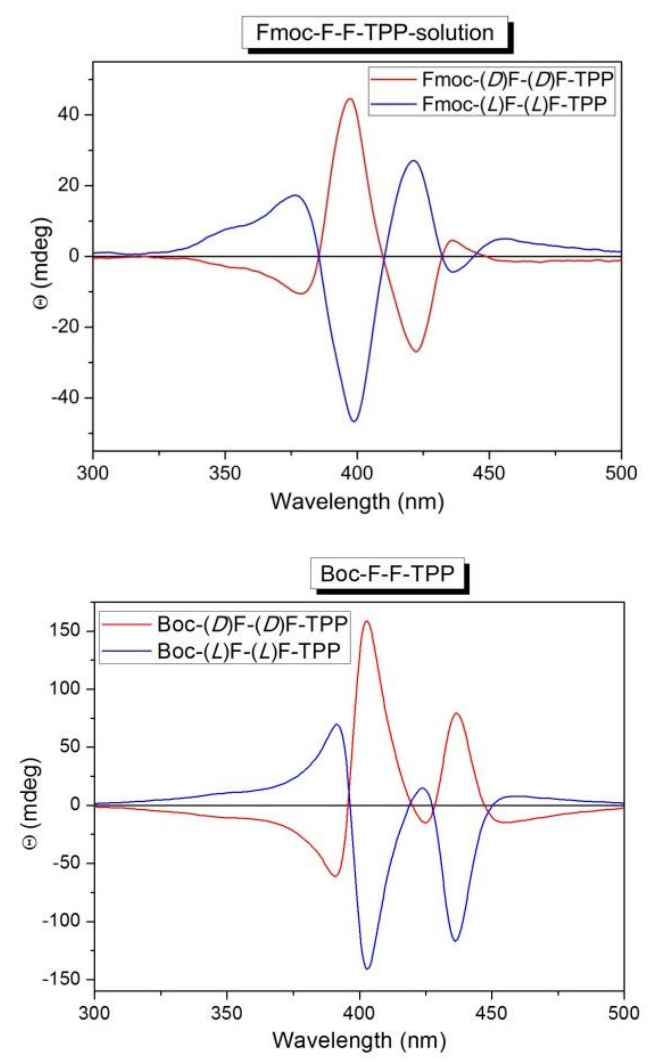

Fig. 4. CD spectra of Fmoc- (Top) and Boc- (Bottom) protected complexes

\section{Conclusion}

We have reported herein diphenylalanine-porphyrin conjugates. All the synthesized compounds self-assemble upon drying into porous spheres with tunable size dependent on the solvent conditions. These compounds selfassemble in nonpolar solvents giving very intense $\mathrm{CD}$ signals; the protecting group influences the profile of the $\mathrm{CD}$ spectra. At a certain concentration film formation was observed on the walls of the cuvette, this film also gives $C D$ signals. This is to our knowledge the first artificial antenna system which can be switched reversibly between a monomeric and a large aggregate by slightly changing only the solvent's polarity. 


\section{References}

[1] Balaban, T. S. "Tailoring Porphyrins and Chlorins for Self-Assembly in Biomimetic Artificial Antenna Systems", Acc. Chem. Res. 38 (2005): 612-623.

[2] Gazit, E. "Self-assembled peptide nanostructures: the design of molecular building blocks and their technological utilization", Chem. Soc. Rev. 36 (2007): 1263-1269.

[3] Charalambidis, G.; Kasotakis, E.; Lazarides, T.; Mitraki, A.; Coutsolelos, A. G. "Self-Assembly Into Spheres of a Hybrid Diphenylalanine-Porphyrin: Increased Fluorescence Lifetime and Conserved Electronic Properties", Chem. Eur. J. 17 (2011): 7213-7219. 